\title{
Comment on "Deep learning computer vision algorithm for detecting kidney stone composition"
}

\author{
ZhiCan $\mathrm{He}^{1} \cdot$ LingYue $^{\mathrm{A}}{ }^{1} \cdot$ ZhengLin Chang $^{1} \cdot$ WenQi Wu${ }^{1}$
}

Received: 16 March 2020 / Accepted: 23 March 2020 / Published online: 1 April 2020

(c) Springer-Verlag GmbH Germany, part of Springer Nature 2020

\section{Dear Editor,}

With great interest, we read the article written by Black et al. [1]. The study showed that deep learning (DL) computervision algorithm can be used to detect the stone composition from digital image, which could provide real-time feedback for laser settings. The high potential of the novel technology in AI deserves the attention of urologists. However, some matters are worth considering.

Firstly, DL technology only allows the prediction of stone composition during operation. However, to get the result preoperatively is likely to be more directly useful for planning therapeutic protocol. For example, uric acid (UA) stone is deemed as the unique stone type that could be treated by oral medical therapy [2]. Also, struvite stone as a type of infection stones is necessary for an initial regimen of antibiotics preoperatively [3], which could effectively decrease the occurrence of complications such as postoperative fever, SIRS and urosepsis.

Secondly, we think there is still a lot of room for improvement in predicting accuracy. A study by Zhang et al. [4] has shown that dual-source dual-energy CT (DSDECT) with excellent accuracy in predicting common chemistry composition. The accuracy (UA 97.5\%, calcium oxalate $93.8 \%$, cystine $93.8 \%$ ) is higher than that of DL technology (UA $94 \%$, COM $90 \%$, cystine $75 \%$ ), especially for cystine stone. Most patients may tend to ignore the radiation exposure to choose the technology with more accurate outcome.

Thirdly, as we know, the results of infrared spectroscopy are limited by the sampled stone, and even the most sophisticated dual-energy CT-DSDECT could only predict

WenQi Wu

wwqwml@163.com

1 Guangdong Key Laboratory of Urology, Department of Urology, Minimally Invasive Surgery Center, Guangzhou Institute of Urology, The First Affliated Hospital of Guangzhou Medical University, Kangda Road 1\#, Haizhu District, Guangzhou 510230, Guangdong, China the major stone composition of stones. So if DL technology could record all layers of the stones in different locations, a more comprehensive individual database about mixed stone compositions could be subsequently established, which might lead to a deeper understanding of the etiology. At present DL technology in this study was applied only for the pure stones. Thus, improving the predicting abilities for mixed stones and the stones with rare composition is the most important item in the future research direction of DL technology.

We agree with the author that DL algorithm is a very promising technology with forward thinking. The concept raised by the author that integrating endoscopic and laser systems by DL technology has its own broad prospects in AI. We hope that our comment will aid further improvement of the technology. We look forward to more high-quality research, as a more accurate DL technology would further promote the realization of AI.

\section{References}

1. Black KM, Law H, Aldoukhi A et al (2020) Deep learning computer vision algorithm for detecting kidney stone composition. BJU Int. https://doi.org/10.1111/bju.15035

2. Ngo TC, Assimos DG (2007) Uric acid nephrolithiasis: recent progress and future directions. Rev Urol 9(1):17-27

3. Flannigan R, Choy WH, Chew B et al (2014) Renal struvite stones-pathogenesis, microbiology, and management strategies. Nat Rev Urol 11(6):333-341

4. Zhang G, Sun H, Xue H et al (2016) Prospective prediction of the major component of urinary stone composition with dual-source dual-energy CT in vivo. Clin Radiol 71(11):1178-1183

Publisher's Note Springer Nature remains neutral with regard to jurisdictional claims in published maps and institutional affiliations. 